\title{
Factors affecting context specificity of appetitive conditioned responding
}

Citation for published version (APA):

Maes, J. H. R., Havermans, R. C., \& Vossen, J. M. H. (2000). Factors affecting context specificity of appetitive conditioned responding. Behavioural Processes, 48, 48-48. https://doi.org/10.1016/S03766357(99)00076-5

Document status and date:

Published: 01/01/2000

DOI:

10.1016/S0376-6357(99)00076-5

Document Version:

Publisher's PDF, also known as Version of record

Document license:

Taverne

Please check the document version of this publication:

- A submitted manuscript is the version of the article upon submission and before peer-review. There can be important differences between the submitted version and the official published version of record.

People interested in the research are advised to contact the author for the final version of the publication, or visit the DOI to the publisher's website.

- The final author version and the galley proof are versions of the publication after peer review.

- The final published version features the final layout of the paper including the volume, issue and page numbers.

Link to publication

\footnotetext{
General rights rights.

- You may freely distribute the URL identifying the publication in the public portal. please follow below link for the End User Agreement:

www.umlib.nl/taverne-license

Take down policy

If you believe that this document breaches copyright please contact us at:

repository@maastrichtuniversity.nl

providing details and we will investigate your claim.
}

Copyright and moral rights for the publications made accessible in the public portal are retained by the authors and/or other copyright owners and it is a condition of accessing publications that users recognise and abide by the legal requirements associated with these

- Users may download and print one copy of any publication from the public portal for the purpose of private study or research.

- You may not further distribute the material or use it for any profit-making activity or commercial gain

If the publication is distributed under the terms of Article $25 \mathrm{fa}$ of the Dutch Copyright Act, indicated by the "Taverne" license above, 


\title{
BeHAVIOURAL PROCESSES
}

\section{Factors affecting context specificity of appetitive conditioned responding}

\author{
J.H.R. Maes *, R.C. Havermans, J.M.H. Vossen \\ Department of Comparative and Physiological Psychology, University of Nijmegen, P.O. Box 9104, \\ $6500 \mathrm{HE}$ Nijmegen, The Netherlands
}

Received 14 July 1999; received in revised form 28 September 1999; accepted 4 October 1999

\begin{abstract}
The present experiment contributes to the identification of factors affecting magnitude of context specificity of simple appetitive conditioned responding. Rats were first trained to associate an auditory and a visual stimulus with food. Each of these stimuli was consistently presented in a distinctive environmental context. Groups of rats differed only in the number of conditioning trials. At test, all groups received trials on which each of the stimuli was presented either in the same context as used during training, or in the different context. Rats made significantly fewer food-magazine visits on different-context trials than on same-context trials only under the conditions that the stimulus tested was the auditory stimulus, which generally elicited a stronger conditioned response (CR) than did the visual stimulus, and the animals had received a relatively small number of conditioning trials. Apparently, magnitude of context specificity is affected by factors determining the strength of the appetitive conditioned response to the target stimulus. (C) 2000 Elsevier Science B.V. All rights reserved.
\end{abstract}

Keywords: Appetitive conditioning; Context specificity; Rat

\section{Introduction}

Previous research examining context specificity of conditioned responding after simple conditioning has generated mixed results. Some researchers found that, after consistent conditioning of a target stimulus, testing that stimulus in an environmental context different from that used during conditioning yields a conditioned response (CR)

\footnotetext{
* Corresponding author. Tel.: + 31-24-3612544; fax: + 3124-3616066.

E-mail address: maes@nici.kun.nl (J.H.R. Maes)
}

that is weaker than that observed when testing occurs in the conditioning context. This context specificity has been found in both appetitive (e.g. Hall and Honey, 1989; Honey et al., 1990) and aversive (e.g. Bonardi et al., 1990; Hall and Honey, 1990) preparations. However, other researchers, mostly using aversive conditioning procedures, failed to do so (e.g. Bouton, 1993; Lovibond et al., 1984).

Demonstrations of context specificity in procedures that do not imply an explicit discrimination procedure are important for both theoretical and practical reasons. Context specificity may reflect 
instances of contextual control that are based upon the potential of context cues to signal, or help to retrieve, an association between the target conditioned stimulus (CS) and the unconditioned stimulus (US), rather than upon more simple context-US or context-CS associations. Some authors (e.g. Bouton, 1994) suggest that this higher-order contextual control, also called occasion setting, occurs exclusively as a result of explicit discrimination training, whereby a target CS acquires more than one 'meaning' in terms of its being (non-)reinforced by a US. However, others deny that stimulus ambiguity is a necessary condition (e.g. Bonardi et al., 1990). At a practical level, results of research on context specificity of conditioning are of relevance for clinical and educational settings (e.g. Bouton, 1988).

Given this theoretical and practical significance, and the mixed results of previous research, it is important to establish the conditions responsible for reliable context specificity of conditioned responding that is based upon contextual occasion setting. Previous research suggests that the number of conditioning trials is of marked importance in this respect. Bonardi et al. (1990), using a flavor-aversion preparation, found context specificity after a multitrial conditioning procedure, but not after a one-trial procedure. However, Hall and Honey (1990) reported just the opposite pattern of results in a conditioned emotional response (CER) procedure with a shock as US.

It remains to be seen whether and how this variable affects context specificity in appetitive conditioning.

Aside from this acquisition factor affecting associative strength, performance variables may also be important. In an unpublished study we used somewhat different conditioning parameters as employed in the present experiment. We found significant context specificity when assessing CR strength immediately after the target CS (post-CS responding). However, no such specificity was found on the basis of an evaluation of CR strength during the CS (CS responding). Post-CS responding was stronger than CS responding, suggesting temporal control of condi- tioned responding (see also, e.g. Maes and Vossen, 1992; Cole et al., 1995). It may well be that the sensitivity to uncover context specificity is maximal when behavioural testing is performed at a moment closely corresponding with the US delivery time during conditioning. Relatedly, in pilot studies, we consistently observed a difference in CR strength as a function of the nature of the CS (visual or auditory). This difference might reflect differences in the form of the CR, rather than differences in CS-US associative strength (see also Holland, 1977). Nature of CS, and corresponding CR strength, may then be viewed as another performance factor. Alternatively, the difference in CR strength might reflect differences in stimulus salience. If so, the nature of the CS constitutes another factor affecting conditioned response acquisition (e.g. Rescorla and Wagner, 1972). In either case, because of its effect on CR strength, the nature of the CS can be viewed as another factor potentially affecting magnitude of context specificity.

The purpose of the present experiment was to examine the effect of these acquisition and performance factors on magnitude of context specificity of simple appetitive conditioning in rats. Specifically, we used a design modelled on that used by Hall and Honey (1989), Honey et al., (1990). First, a visual and an auditory stimulus were trained as reliable predictors for food. Each of these stimuli was presented in a separate, distinctive environmental context. Experimental groups differed only in the number of training trials. Different groups received 12, 24, 36 or $48 \mathrm{CS}-\mathrm{US}$ conditioning trials. At test, each CS was presented both in the same context as used during conditioning and in the other context. This allowed us to compare, within subjects, CR strength on trials on which each of the CSs was presented in its original training context (same trials), and in a context different from the training context (different trials), to assess context specificity. The present design equates the number of USs presented in each of the test contexts. Moreover, we also assessed CR strength by examining number of food-magazine visits both during and immediately after each CS presentation. 


\section{Materials and methods}

\subsection{Subjects}

Thirty-two male Wistar rats served as subjects. They were 2-3 months old and had a mean free-feeding body weight of $195 \mathrm{~g}$. Animals were individually housed in Plexiglas cages with a bedding of wood shavings. These cages were located in a colony room that was on a $12: 12$-h light dark cycle (lights out at 08:00 h). Experimental sessions were run on week-days between 09:00 and 17:00 h. The rats were maintained at $85 \%$ of their free-feeding body weight by restricted daily feeding. The subjects had free access to water in the home cage.

\subsection{Apparatus}

Two sets of four boxes each were used for conditioning and testing. Each of the boxes of the first set measured $24.5 \times 25.0 \times 20.0 \mathrm{~cm}^{3}$ and consisted of a front wall, back wall, and a ceiling made from clear Plexiglas. The two side walls were aluminium and the floor consisted of an aluminium grid with $0.5 \times 0.5 \mathrm{~cm}^{2}$ holes. Centred on the left-side wall was a recessed food magazine measuring $5 \times 5 \times 3 \mathrm{~cm}^{3}$. The base of the food magazine was at the same level as the grid floor. Delivery of a single 45-mg food pellet (Campden Instruments, Sileby, Loughborough, UK) into the magazine served as the US. The magazine contained an infrared emitter and sensor that enabled registration of food magazine visits. The box was permanently lit by three light-emitting diodes (LEDs; Sharp Corporation, Electronic Components Group, Type no. LT-9510E; $565 \mathrm{~nm} ; 65$ mcd), and by three $24-\mathrm{V}, 2.8-\mathrm{W}$ light bulbs located behind a white Plexiglas disk (diameter: 1.6 $\mathrm{mm}$ ), except during presentation of the 'dark' CS (switching off of the LEDs and light bulbs). One LED was fixed to the centre of the ceiling; the other two LEDs were fixed to the left-side wall, $9.5 \mathrm{~cm}$ above the floor and $7 \mathrm{~cm}$ to the left and to the right of the food magazine. The three light bulbs were mounted in a row (distance between bulbs: $2.5 \mathrm{~cm}$ ) to the top-centre of the left-side wall, $17 \mathrm{~cm}$ above the floor. A 76-dB(A), 6-kHz auditory CS could be presented through two speakers mounted to the left side wall, $1 \mathrm{~cm}$ above the floor and $9 \mathrm{~cm}$ to the left and to the right of the food magazine. Each of the boxes was enclosed in a shell containing a ventilation fan that produced a $70-\mathrm{dB}(\mathrm{A})$ background noise. The boxes and shells were located in a room that was dimly illuminated by a single red fluorescent strip light.

Each of the boxes of the other set was positioned in a different corner of the experimental room and was identical to the boxes just described, except for the following. The floor consisted of a wooden board $\left(9.5 \times 4.0 \mathrm{~cm}^{2}\right)$ placed in front of the food magazine. The remaining part of the floor was composed of 3-mm stainless-steel rods that were spaced $1.3 \mathrm{~cm}$ apart. The right-side wall contained a speaker (not used) and was composed of the same type of rods as also used to make part of the floor. A plastic ball (diameter: $3.5 \mathrm{~cm}$ ) was fixed to the centre of the floor. Finally, a distinctive odour cue was presented by $\sim 1 \mathrm{~g}$ of Vicks Vaporub, which was applied to a piece of paper towel and placed inside the shell. The auditory CS was presented using the same tone generator as used for presenting the tone in the other set of boxes. The dark stimulus was created as described before. In this manner, it was very likely that each of the CSs was perceived as an identical stimulus in the two types of boxes.

\subsection{Procedure}

Matched on the basis of body weight, the animals were divided into four groups $(n \mathrm{~s}=8)$.

\subsubsection{Magazine training}

All animals first received two 35-min sessions. One session was performed in the morning, between 09:00 and 12:00 $\mathrm{h}$; the other session in the afternoon, between 14:00 and 17:00 h. Each session consisted of the presentation of ten USs according to a variable time 3-min schedule. Two food pellets were present in the food magazine at the start of the first session.

For one half of the subjects in each group, the first type of box described above, hereafter referred to as context 1 , was used in the morning 
session, whereas the second type of box, context 2 , was used in the afternoon session. The other half of the rats was first trained in context 2 and then in context 1.

Groups differed in the timing of magazine training. The rats of group 8 (the group's name refers to the number of conditioning sessions; see hereafter) were trained on day 1 . Those of groups 6,4 and 2 received the magazine training, respectively, 2, 4, 6 and 8 experimental (i.e. week-)days after the magazine training for group 8 .

\subsubsection{Conditioning}

Two 35-min sessions were performed on each experimental day of the conditioning phase: a morning session and an afternoon session. These sessions were performed at approximately the same time as described for the magazine training. The first daily session was performed in context 1 for one-half of the subjects in each group and in context 2 for the other half, whereas the second session was performed in the alternative context. During each session, each of six 10-s presentations of one of the CSs was followed by the US (termination of the CS coincided with the delivery of a food pellet). The first CS-US conditioning trial consistently started 5 min after onset of the session. Thereafter, the interval between US delivery and CS onset was variable around a mean of 5 min (range: 3-6 min). For one half of the animals receiving the morning session in context 1 , the dark CS was conditioned on the first session and the tone CS on the second, whereas the reverse was true for the other half. The same held for the animals receiving the morning session in context 2.

All groups received the first pair of conditioning sessions one day after magazine training but differed in the total number of conditioning days. The rats of groups 2, 4, 6 and 8 received, respectively, 2, 4, 6 and 8 conditioning sessions with each CS. Because magazine training started at different points in time for the different groups, all animals started the test phase at the same day.

\subsubsection{Testing}

Testing was initiated the day after the last conditioning day and consisted of a single morn- ing and afternoon session. These sessions were identical to those of the conditioning phase, except for the following. On each session, the dark and tone CSs were alternately presented, for a total of three presentations of each CS. CS presentations were all nonreinforced. Order of trial type was counterbalanced within groups, such that for one half of the rats the first trial of each test session always was the visual CS, whereas it was the auditory CS for the other half. The nature of the first trial (same or different trial) was also counterbalanced within groups. This test implies three nonreinforced presentations of each of the visual and auditory CSs in both the original training context (same trials) and in the alternative context (different trials).

\subsection{Behavioural measurement and statistical analysis}

During training, we measured the number of magazine visits during each 10 -s CS presentation (CS responding) and during each 10 -s period immediately preceding each CS (pre-CS responding). At test, we also measured the number of visits during the $10 \mathrm{~s}$ that immediately followed each CS (post-CS responding). A post-CS response measure was not used in the training phase because here visits could be triggered by the sounds elicited by the delivery of a food pellet and, thus, do not necessarily reflect the type of associative learning of primary interest. Pilot experiments implied that changes in post-CS responding reflect associative learning as much as do changes in CS responding. For instance, prior to conditioning, termination of each of the stimuli used in the present experiment does not elicit magazine responding on its own. It is only in the course of conditioning that post-CS food-magazine response frequencies start to increase, as assessed by 'probe' trials without reinforcement (i.e. in the absence of sounds produced by the pellet feeder).

Response frequencies at test were subjected to analyses of variance (ANOVAs) with group as between-subjects factor, and stimulus (dark versus tone $\mathrm{CS}$ ), period (CS versus post CS) and trial type (same versus different) as within-subjects factors. Significant interactions involving the trial 
type factor were examined further by simple main effect analyses. The error term and degrees of freedom in these analyses were derived from the overall analysis (Winer, 1971, Ch. 7). The rejection criterion was set at $P<0.05$ throughout.

\section{Results}

\subsection{Conditioning}

Pre-CS response frequencies were low throughout conditioning. The overall mean frequency was 0.4 for group 8 , and 0.5 for each of the remaining groups. There were no differences between groups and CS types. Hereafter, we will focus on CS responding only.

Fig. 1 displays the mean number of magazine visits during CS presentations for each of the

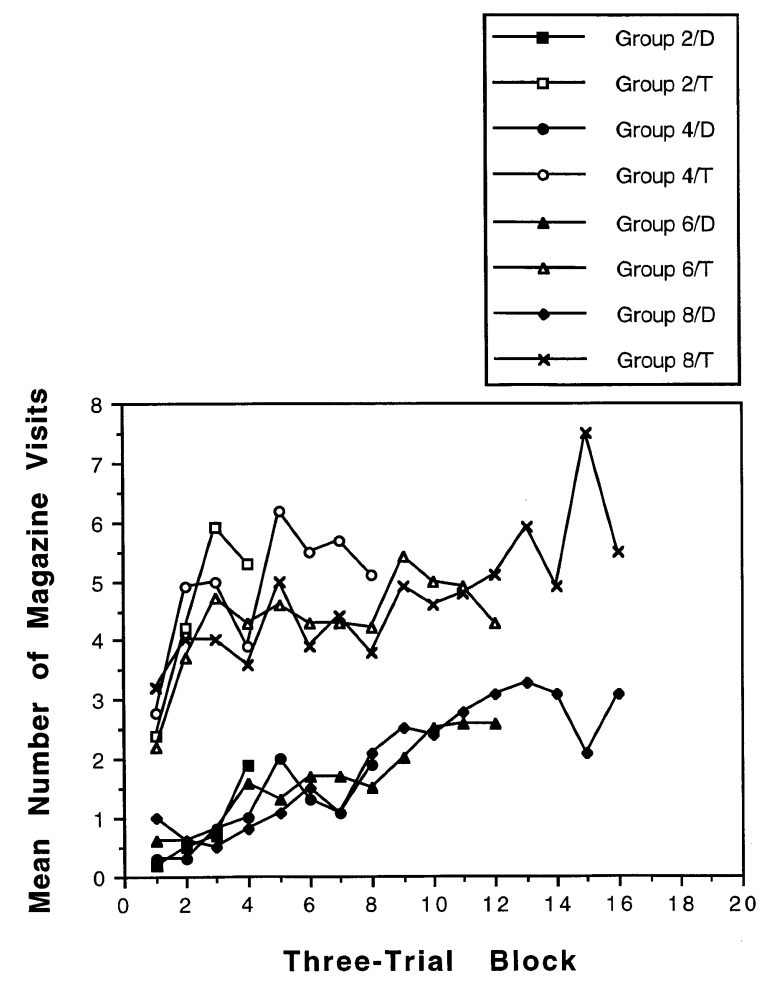

Fig. 1. Mean number of magazine visits as a response to the dark (D) and tone (T) CS across blocks of three conditioning trials. Groups differed in the total number of conditioning blocks they received. groups across three-trial training blocks. Each group responded more during the tone than during the dark stimulus. Furthermore, in each group, there was a gradual increase in response frequency for both CSs (smallest $F(15,105)=$ 2.35). This reflects progressive conditioning. However, at the final block of training for each group, the response frequency did not seem to differ much among groups for each of the CSs.

A group $\times$ CS type (tone versus dark stimulus) ANOVA was performed on the response frequencies observed during the final training block for each of the groups. This analysis only revealed a significant effect of CS type $(F(1,28)=52.86$, other $F \mathrm{~s}<1)$, reflecting more responding during the tone than during the dark stimulus in each of the groups.

\subsection{Testing}

Responding during the pre-CS periods was virtually absent in all groups on each of the test sessions. The overall mean number of visits was only 0.2 and there were no significant differences between groups. We will focus on CS and post-CS responding hereafter.

Mean CS and post-CS response frequencies during same and different trials of the test sessions are displayed in Figs. 2 and 3, respectively, separated for group and CS type. Under the present nonreinforced test conditions, a difference was visible with respect to overall associative strength as a function of number of training trials, especially for the dark CS: the more training trials, the more frequent was responding during $\mathrm{CS}$ and post-CS periods. As observed also during training, rats generally responded less frequently on dark-CS than on tone-CS trials. More interestingly, for group 4 and especially group 2, responding during CS and post-CS periods was more frequent on same trials than on different trials. There was no large and consistent difference in response frequency between trial types in the other two groups.

A group $\times$ stimulus $\times$ period $\times$ trial type ANOVA on the data depicted in Figs. 2 and 3 revealed a significant main effect for group $(F(3$, $28)=3.06)$, stimulus $(F(1,28)=56.77)$, and trial 


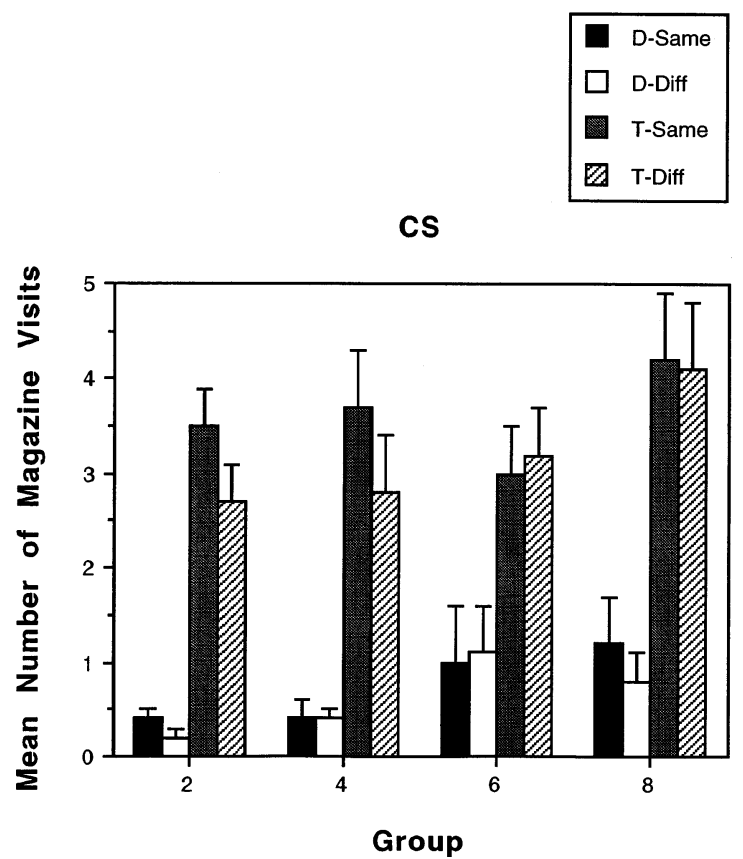

Fig. 2. Mean number of magazine visits ( \pm S.E.M.) as a response to the dark (D) and tone (T) CS during the tests. CSs were presented either in the same context as used during conditioning (same) or in a different context (diff). Groups differed in the total number of conditioning sessions they had received previously.

type $(F(1,28)=5.18)$. Subsequent post hoc analyses revealed that the rats of group 2 made fewer magazine visits than did the rats of group 8 (Newman-Keuls, $P<0.05$ ). None of the other differences among groups was significant. This pattern reflects an increase in associative strength with increasing number of training trials. The significant main stimulus and trial type effects reflect an overall higher response frequency on tone-CS than on dark-CS trials, and on same-context trials than on different-context trials. The following interactions were also significant: group $\times$ trial type $(F(3,28)=6.12)$, stimulus $\times$ period $(F(1,28)=98.09)$, and group $\times$ stimulus $\times$ period $\times$ trial type $(F(3,28)=3.15)$. The latter interaction is of primary interest for present purposes and was investigated further by analyses of the simple main effect of trial type at each possible combination of group, stimulus, and period. These analyses revealed that the rats of group 2 responded significantly more on same-context trials than on different-context trials when examining tone-CS and post-tone-CS responding $(F(1$, $56)=7.29)$ and $F(1,56)=22.64$, respectively). The rats in group 4 also responded significantly more on same-context than on different-context trials when examining CS responding $(F(1,56)=$ $7.29)$, but not when examining post-CS responding $(F(1,56)=1.33)$. None of the other analyses revealed significantly more responding on samecontext than on different-context trials. In fact, the rats in group 6 responded more on differentcontext than on same-context trials during the post-tone-CS period $(F(1,56)=4.24)$; the same was true for the rats in group 8 during the postdark-CS period $(F(1,56)=8.00)$.

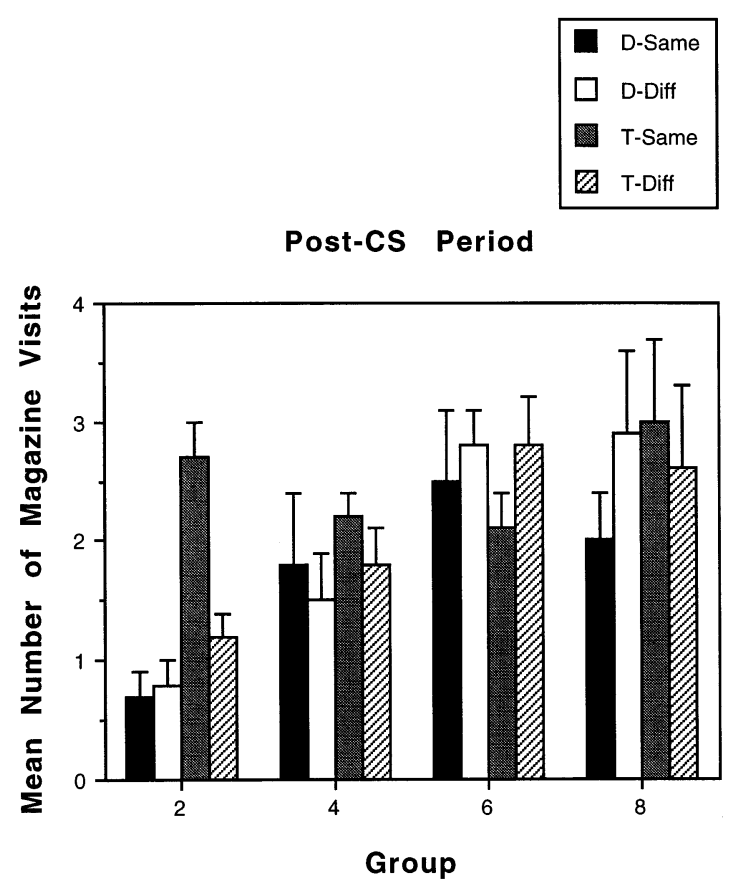

Fig. 3. Mean number of magazine visits $( \pm$ S.E.M. $)$ in the 10 -s period immediately after presentation of the dark (D) and tone (T) CS during the tests. CSs were presented either in the same context as used during conditioning (same) or in a different context (diff). Groups differed in the total number of conditioning sessions they had received previously. 


\section{Discussion}

The present experiment identifies two factors that affect magnitude of context specificity of conditioned responding after simple appetitive conditioning. First, context specificity appeared after a moderate amount of training, but was not seen after more extended training. Second, evidence for context specificity was obtained with regard to the auditory CS (at least in two of the four groups), but not for the visual CS.

It is not possible to account for the absence of context specificity regarding the tone CS in groups 6 and 8, and with respect to the dark CS in all groups, simply in terms of floor- and ceiling effects. The data suggest that, generally, there was ample opportunity to detect differences in response frequencies between trial types on CS and/ or post-CS periods for both CS types. In this respect, it is also noteworthy that we continued testing after the first two test sessions (not reported), but still failed to uncover context specificity in groups 6 and 8 .

A factor that we initially hypothesised would also clearly affect magnitude of context specificity is the timing of behavioural assessment. On the basis of unpublished experiments, we expected context specificity to be most profound when assessing CR strength at a time that corresponds with the US-delivery time during conditioning, namely the post-CS period. Although it is true that group 2 exhibited the strongest difference between same-context and different-context trials during the post-tone periods, group 4 displayed significant context specificity primarily during tone periods. Thus, in the present experiment we did not find consistent evidence for more marked context specificity for post-CS responding than for CS responding. The fact that we did find the indicated difference in magnitude of context specificity in previous studies may be due to a difference in CS duration between experiments. In the present experiments, CS duration was only $10 \mathrm{~s}$, whereas it was $30 \mathrm{~s}$ in the previous studies. The latter duration may promote a larger sensitivity for post-CS response measures than for CS measures. Still, collectively, the available data do suggest that it is useful to collect both CS and
post-CS response measures in experiments on context specificity.

An important question that remains to be addressed is whether or not the context specificity found for the tone CS in groups receiving moderate training was based upon the training context functioning as occasion setter for a tone $\rightarrow$ food relationship (or, perhaps, for a tone $\rightarrow$ magazine response $\rightarrow$ food relationship). An account of this specificity that does not require the notion of occasion setting is that the tone CS was perceived differently in the two contexts. In other words, the stronger $\mathrm{CR}$ in the same than in the different context may simply have been based upon generalisation decrement. Although it is difficult to entirely rule out this explanation, it is less likely given the following. First, the tone was produced by one and the same tone generator in the two contexts, which is a condition that minimises the likelihood of different perceptions of the tone CS. Second, early research on generalisation of CRs suggests that such generalisation decreases with increasing number of (non-)reinforced test trials (e.g. Razran, 1949; Wickens et al., 1954; Desiderato, 1964). In the present research this would imply that the degree of context specificity (i.e. the inverse of generalisation) is a function of test-trial number: all other things being equal, magnitude of context specificity should increase with increasing number of test trials (within limits because of extinction). However, ANOVAs using CS and post-CS response frequencies that included a test trial factor revealed a significant main effect for this factor, reflecting a gradually decreasing CR in the course of testing. Importantly, this factor did not significantly interact with any other factors of primary interest (group and trial type). Third, Bonardi (1992), using a preparation very similar to the present one, found evidence for context specificity in experiments that could more rigorously discard a generalisation-decrement account (see also Honey and Watt, 1999).

Another account that does not demand the notion of occasion setting is that the weaker CR on different-context trials reflects enhanced stimulus processing. For example, tone conditioning might have resulted in the formation of a context-CS association. As a result of this associa- 
tion, the CS may have been processed to a lesser extent in the training context than in the other context upon later presentation (i.e. negative priming; e.g. Wagner, 1981). This allows for the possibility that extinction of the tone CS in the course of testing occurred at a faster rate in the different context (strong CS processing) than in the same context (weak CS processing).

This line of reasoning cannot provide a complete explanation of the context specificity found. This is because it implies that the extent of context specificity is a function of test-trial number, which was not the case in the present experiment (see above). However, one aspect of the present data is consistent with a negative-priming notion: the rats receiving relatively extended training (groups 6 and 8) on some occasions even displayed an increased $\mathrm{CR}$ on different context-trials than on same-context trials. Notably, this effect is similar to that reported by Honey et al. (1993). These authors found this effect to be consistent with Wagner's model.

In fact, a contextual priming notion may be combined with a contextual occasion-setting notion to provide a coherent account of the present results (see also Hall and Mondragón, 1998). Accordingly, CS-US pairings in a distinctive context may prompt two different processes. One is that the context acquires the capacity to signal, retrieve, or gate the CS-US association (occasion setting). The second process is contextual priming as a result of the formation of a context-CS association. The relative strength of each of these two properties of context will determine the outcome of a test manipulating environmental context, as performed in the present experiment. Contextual occasion setting will promote more vigorous responding in a 'same-context' test condition than in a 'different-context' test condition. Instead, contextual priming will encourage weaker responding in the same-context condition than in the different context condition. It is quite conceivable that the strength of a context-CS association (and, thus, of contextual priming), will increase with increasing number of conditioning trials. This, in turn, would imply decreasing context-specificity, and even a 'reversed' context specificity (more responding in different context than in same context), with continued training. These notions might also provide a framework for explaining the difference found in our experiment regarding sensitivity for context specificity as a function of type of stimulus used. Possibly, strength and/or rate of formation of a contextCS association differ for different CS types.

Whatever the merits of these theoretical speculations, the present experiment provides an important empirical contribution to the issue of the conditions determining context specificity. Any theory of context specificity should be able to handle the fact that reliable context specificity may only occur with specific conditioning parameters. The fact that previous research on this subject has generated mixed results may reflect differences in just these parameters.

\section{References}

Bonardi, C., 1992. Occasion setting without feature-positive discrimination training. Learn. Motiv. 23, 343-367.

Bonardi, C., Honey, R.C., Hall, G., 1990. Context specificity of conditioning in flavor-aversion learning: extinction and blocking tests. Anim. Learn. Behav. 18, 229-237.

Bouton, M.E., 1988. Context and ambiguity in the extinction of emotional learning: Implications for exposure therapy. Behav. Res. Ther. 26, 137-149.

Bouton, M.E., 1993. Context, time, and memory retrieval in the interference paradigms of Pavlovian learning. Psychol. Bull. 114, 80-99.

Bouton, M.E., 1994. Conditioning, remembering, and forgetting. J. Exp. Psychol. Anim. Behav. Process 20, 219-231.

Cole, R.P., Barnet, R.C., Miller, R.R., 1995. Temporal encoding in trace conditioning. Anim. Learn. Behav. 23, 144153.

Desiderato, O., 1964. Generalization of acquired fear as a function of CS intensity and number of acquisition trials. J. Exp. Psychol. 67, 41-47.

Hall, G., Honey, R.C., 1989. Contextual effects in conditioning, latent inhibition, and habituation: associative and retrieval functions of contextual cues. J. Exp. Psychol. Anim. Behav. Process 15, 232-241.

Hall, G., Honey, R.C., 1990. Context-specific conditioning in the conditioned-emotional-response procedure. J. Exp. Psychol. Anim. Behav. Process 16, 271-278.

Hall, G., Mondragón, E., 1998. Contextual control as occasion setting. In: Schmajuk, N.A., Holland, P.C. (Eds.), Occasion Setting; Associative Learning and Cognition in Animals. APA, Washington, DC, pp. 199-222.

Holland, P.C., 1977. Conditioned stimulus as a determinant of the form of the Pavlovian conditioned response. J. Exp. Psychol. Anim. Behav. Process 3, 77-104. 
Honey, R.C., Watt, A., 1999. Acquired relational equivalence between contexts and features. J. Exp. Psychol. Anim. Behav. Process 25, 324-333.

Honey, R.C., Willis, A., Hall, G., 1990. Context specificity in pigeon autoshaping. Learn. Motiv. 21, 125-136.

Honey, R.C., Hall, G., Bonardi, C., 1993. Negative priming in associative learning: evidence from a serial-conditioning procedure. J. Exp. Psychol. Anim. Behav. Process 19, 90-97.

Lovibond, P.F., Preston, G.C., Mackintosh, N.J., 1984. Context specificity of conditioning, extinction, and latent inhibition. J. Exp. Psychol. Anim. Behav. Process 10, 360-375.

Maes, J.H.R., Vossen, J.M.H., 1992. One-trial aversive conditioning to contextual cues: effects of time of shock presentation on freezing during conditioning and testing. Bull. Psychon. Soc. 30, 403-406.
Razran, G., 1949. Stimulus generalization of conditioned responses. Psychol. Bull. 46, 337-365.

Rescorla, R.A., Wagner, A.R., 1972. A theory of Pavlovian conditioning. Variations in the effectiveness of reinforcement and nonreinforcement. In: Black, A.H., Prokasy, W.F. (Eds.), Classical Conditioning II: Current Research and Theory. Appleton-Century-Crofts, New York, pp. 6499.

Wagner, A.R., 1981. SOP: a model of automatic memory processing in animal behavior. In: Spear, N.E., Miller, R.R. (Eds.), Information Processing in Animals. Erlbaum, Hillsdale, NJ, pp. 5-47.

Wickens, D.D., Schroder, H.M., Snide, J.D., 1954. Primary stimulus generalization of the GSR under two conditions. J. Exp. Psychol. 47, 52-56.

Winer, B.J., 1971. Statistical Principles in Experimental Design, 2nd ed. McGraw-Hill, New York. 